\title{
Remote monitoring and sophisticated analysis of status data from power generators
}

\author{
Martin Bussas, Philipp Baum \\ TROUT GmbH, Parkstr.28, 34119 Kassel, Germany \\ m.bussas@trout-gmbh.de
}

\begin{abstract}
Selected power generator data, which is exchanged via CAN bus or MODBUS, is picked from the data bus by a Programmable Logical Controller PLC. Together with position data, which is provided by a separate GPS module, it is sent via Uniform Data Protocol UDP using a modem and the Global System for Mobile Communications GSM to a dedicated internet server. On the internet server, the received data is analyzed and stored in a database management system.

The content of the database is processed by a web server and can be accessed user-dependently and password-protected with any browser on personal computers, tablets or smartphones. The layout of the display automatically adjusts to the display capabilities of the terminal. In addition, static data of the device such as operating instructions and spare parts lists can be viewed.

The position data of the device is used for a geographical representation of the power supply on google maps. In addition, if the power supply is a mobile generator, a major change of the position may be used to generate a theft alarm via e-mail or text message.
\end{abstract}

Key words: PLC, GSM, GPS, power generator

\section{Remote Monitoring and Statistical Evaluation Overview}

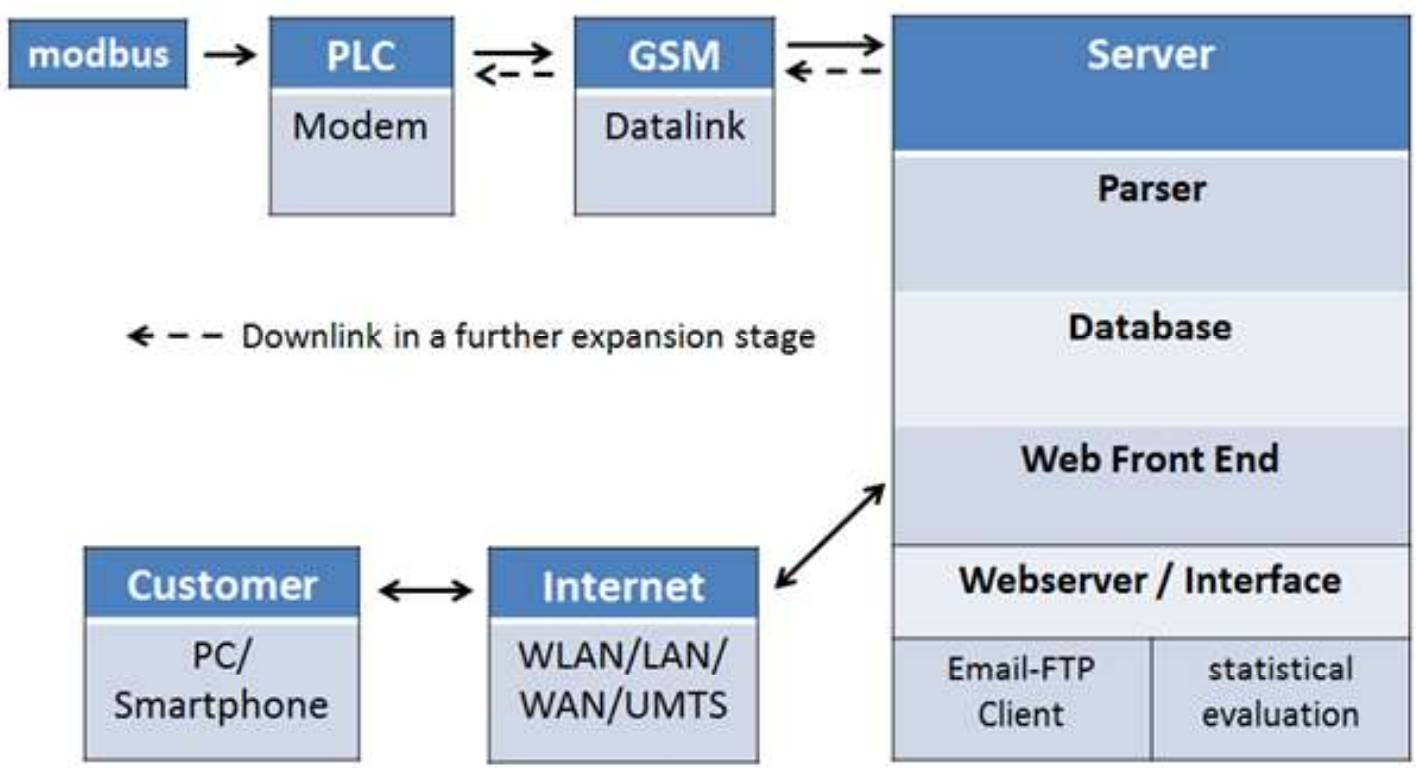

Fig.1. System Structure 


\section{Processing of message and operating data}

The power generator transports the requested operating MOD/CANBUS data to the transmission unit which combines the operating data with further parameters (date, time, position) and creates a data package which is sent to the server.

The power generators are either independent generators (diesel generators, PV plants), power distributors or stores that are integrated into the grid. The data collection corresponds to the respective type.

\section{Web portal}

After logging in with user data and password, all power generators that are assigned to the user and stored in the server's database are displayed. The display arrangement depends on the resolution of the user device.

The button 'Parameter' displays a more detailed view and the user can access the operating data.

\section{Operating modes}

Examples of different operating modes of power generators follow:

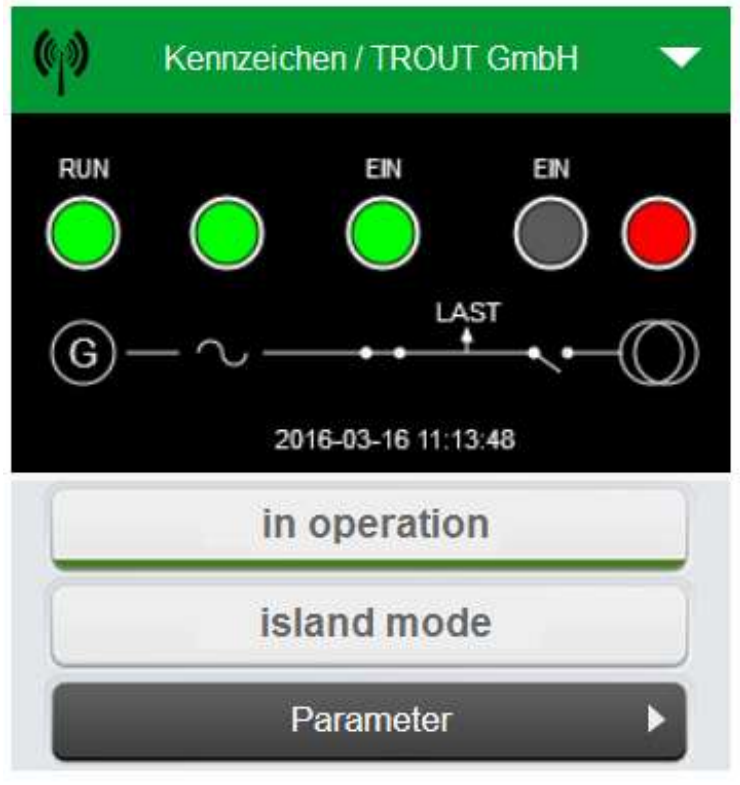

Fig.2.0. Power generator on, grid not available and not connected.

Figure 2.0 shows a fully functional generator in island mode. This means that the generator has no connection to the power grid. As an indication, the switch on the right hand side of LAST (power consumer) is open. The switch on the left hand side of LAST is closed, which means that LAST is supplied with energy by the power generator.

A generator which is out of operation is shown in figure 2.1.

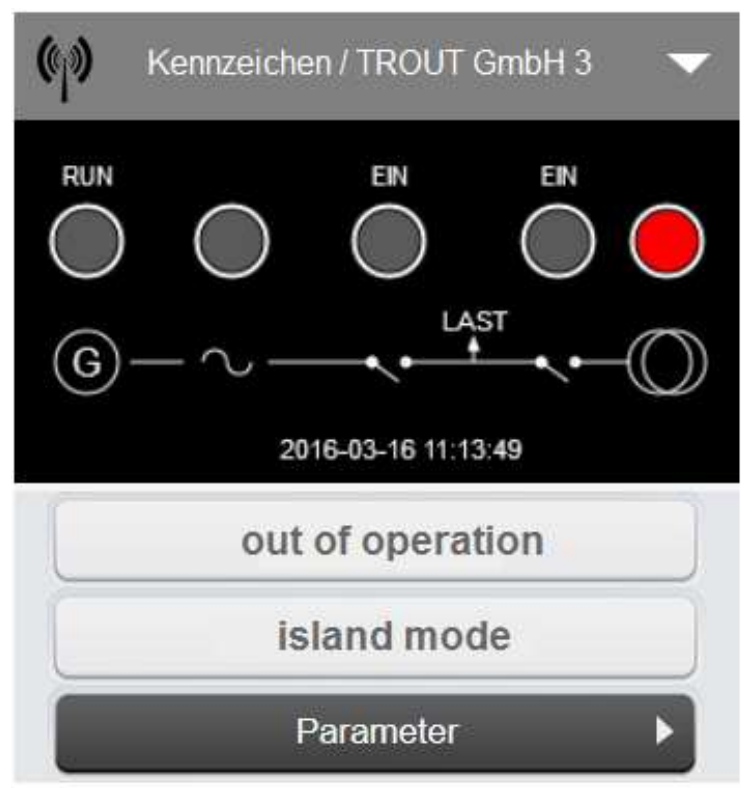

Fig.2.1. Power generator off, grid not available and not connected.

Figure 2.2 shows a generator which is in an unknown state because the data link is broken, which is indicated by the crossed antenna symbol on the upper left. LAST is supplied by the grid. The last data set, before the connection was broken, was transferred at $10: 44$ on the $26^{\text {th }}$ January, which is displayed on the bottom of the black rectangle.

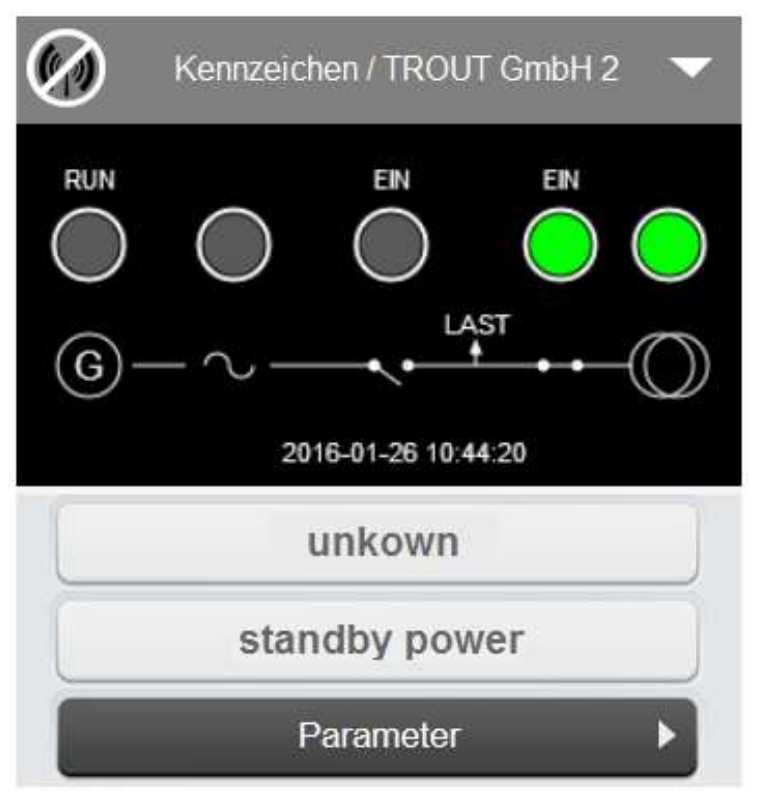

Fig.2.2. No data transfer from power generator, grid available and connected. 

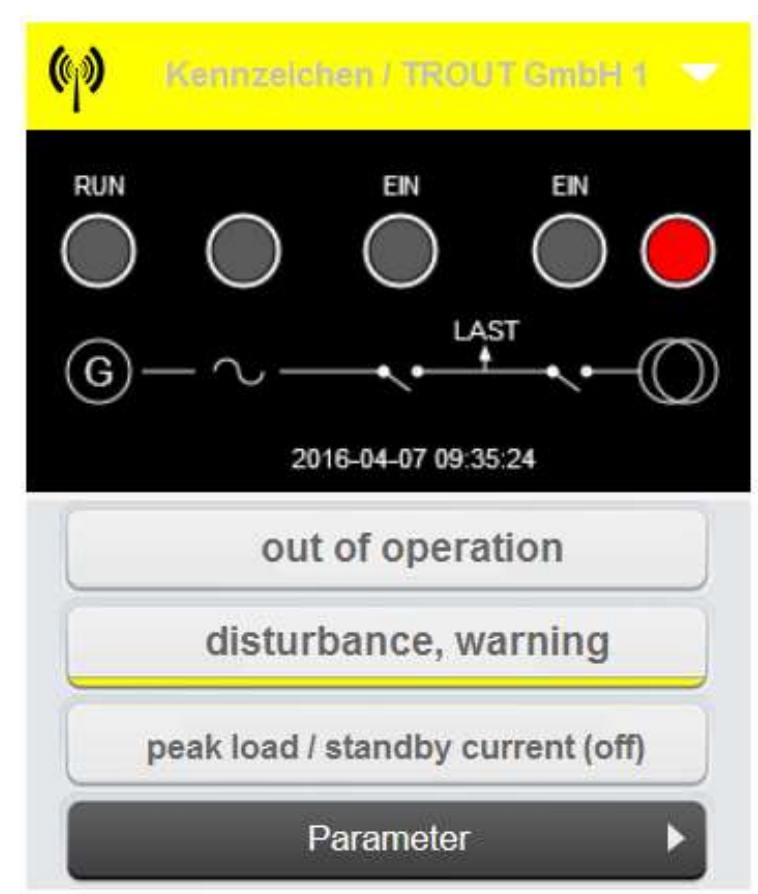

Fig.2.3. Disturbance message and warning from power generator.

A yellow marking indicates that the generator is sending warnings (see figure 2.3). The disturbances reach from problems such as 'fuel level is low' to minor malfunction of the generator.

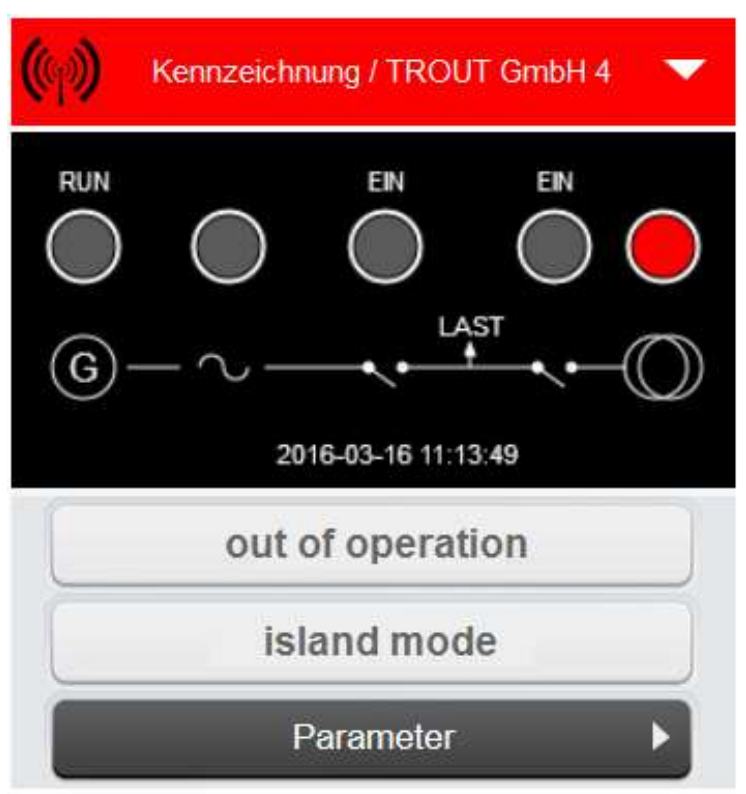

Fig.2.4. Malfunction of power generator, error message sent.
If the generator has a major problem, the marking turns red (see figure 2.4.). The service technician is informed via e-mail or text message. The message is repeated every 24 hours until the cause of the malfunction status is eliminated.

\section{Integration of a GPS module}

The power generator's position can be monitored with an integrated GPS module. Logging on to the monitoring portal, the user can invoke a geographical overview of his power generators. The power generator is visualised on a digital map with a colored marker depending on the condition, and a label. If the power generator is moved from its assigned position, a displacement message may be sent.

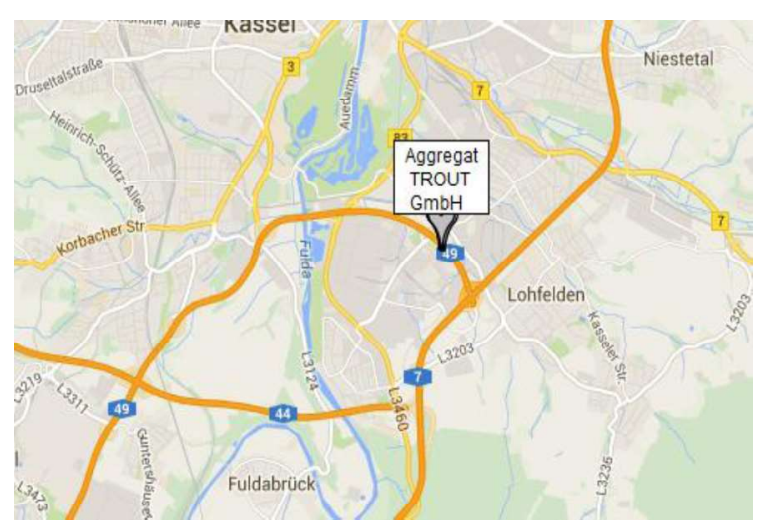

Fig.3. Visualisation of geographic position on a digital map.

\section{Customised adjustment according to customer requirements}

The application offers configuration of the user interface according to the customer requirements including optical adjustments such as background or company logo, as well as menu adaptation.

As the server receives many parameters from the power generator at any time, the user may want to display a selection only. Therefore it is possible to create a personal template. The selected parameters are then displayed in a customised list. Figure 4 shows an example. 


\begin{tabular}{|l|c|}
\hline Parameter \\
\hline Generator voltage [V]: & 396 \\
\hline Generator frequency [Hz]: & 50,9 \\
\hline Mains voltage [V]: & 396 \\
\hline Main frequency [Hz]: & 50,9 \\
\hline Power factor [cos $\varphi$ ] & 1,00 \\
\hline Fuel level [\%]: & 29 \\
\hline Residual term [h]: & 5 \\
\hline Motor temperature [ $\left.{ }^{\circ} \mathrm{C}\right]:$ & 79 \\
\hline Next maintenance $[\mathrm{d}]:$ & 48 \\
\hline
\end{tabular}

Fig. 4. Customised parameter list.

\section{Advanced administration of remote monitoring customers}

Manufacturers or distributers of power generators may offer their customers remote monitoring as an additional service. The manufacturer or distributer receives administrator right for user administration in order to add users and assign power generators to them. In the following the users customise the handling of the operating data themselves.

\section{Statistical evaluation of the operating data}

The module for statistical evaluation creates a collection of selected operating data using the historical data of the power generator from the database on the server (work, fuel consumption, oil pressure). The data collection can be displayed graphically in many different views.

For instance, the generator's work may be shown over a period of time. Furthermore, different operating data can be combined in one diagram, to examine potential dependences such as work and fuel consumption.

The module for statistical evaluation further offers to the user the output of specific profitability factors of the power generators, such as running costs and efficiency. If a previously set value range is not achieved, the application will display and send a warning message.

\section{Plots}

Figure 5 shows power together with fuel level of a power generator. The fuel fillings and daily consumption can easily be seen. Due to measurement inaccuracies, time intervals occur during which the fuel level stays on one level, unaffected by operation. Similarly, disturbances emerge that interrupt the even curve progression (see fig. 5: 18:00 to 22:00). [1][2]

These irregularities complicate the statistical processing and subsequent evaluation of the operating data. The application addresses this problem and compensates potential disturbances by catching such artefacts with integrated filters. Fig. 6 shows the effect of one such filter.

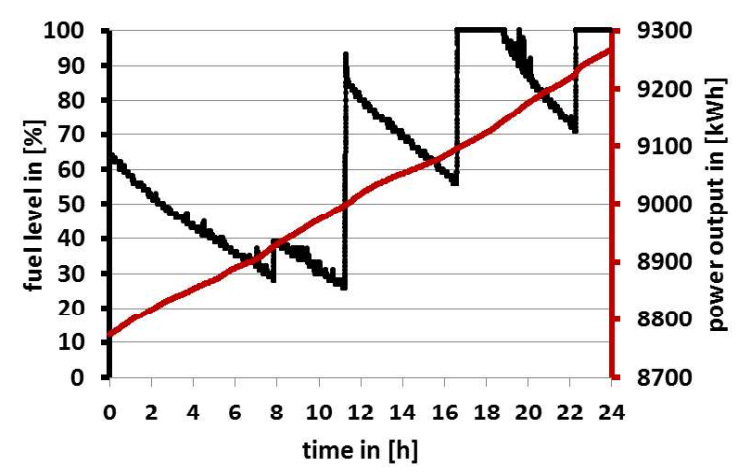

Fig.5. Power and fuel vs. time

After being processed by the filter, the data is analysed more specifically together with the technical parameters of the power generator.

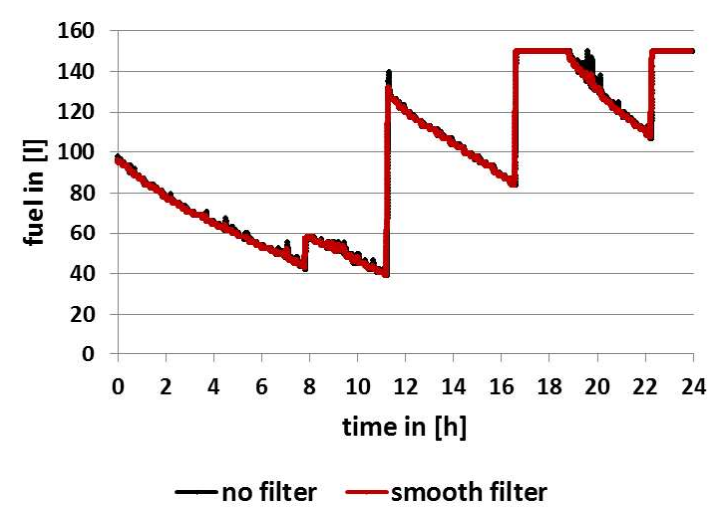

Fig.6. Filter options

A straightforward example is fuel consumption per kilowatt hour. The application calculates the values and displays them graphically for the user (see Figure 7). 


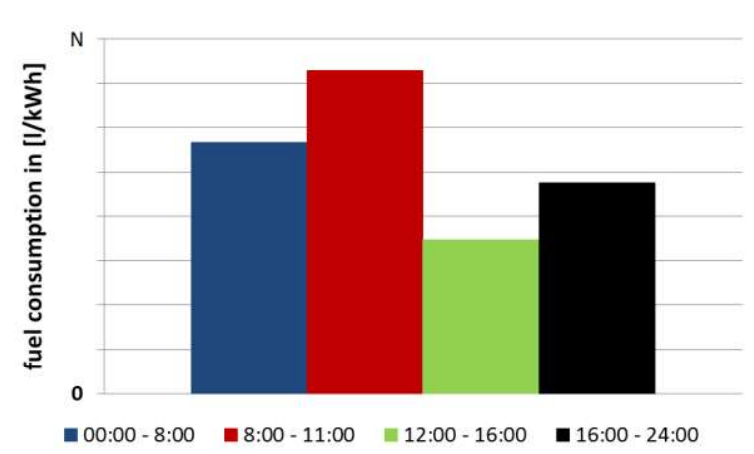

Fig.7. Fuel consumption per kilowatt hour.

The module for statistical evaluation compares the operating data with manufacturer specifications and generates a protocol of deviations. Over longer periods of time, for example, decreasing efficiency or increasing fuel consumption can be observed.

The module detects trends and may send messages to the manufacturer, distributer or service technician.

In addition, the operating cost may be calculated. This includes variable costs, such as fuel price, and fixed costs such as rent, depletion and maintenance.

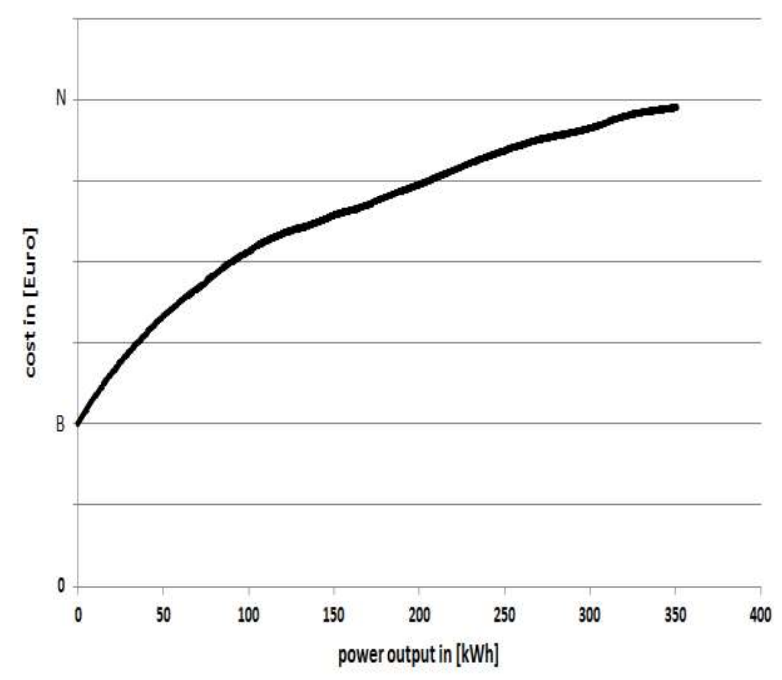

Fig. 8. Cost per kilowatt hour and day.

The user is free to combine any single parameters with each other. As the example in figure 9 shows.

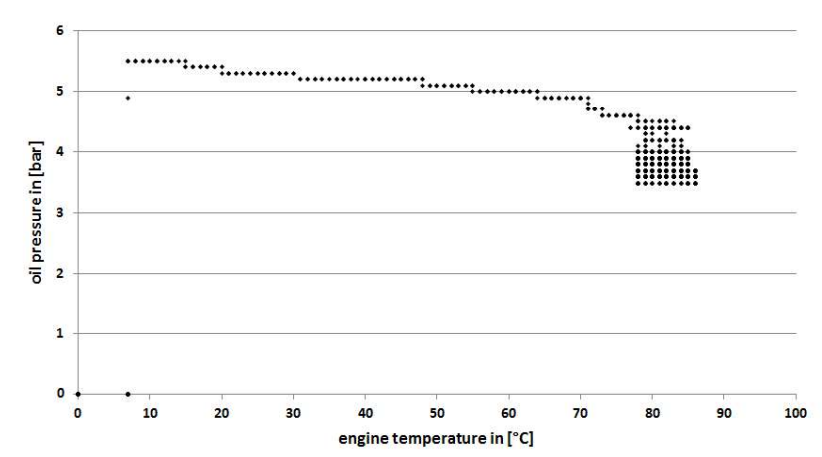

Fig.9. Oil pressure vs. motor temperature.

The module for statistical evaluation uses collected operating data, manufacturer specifications and the recorded malfunctions. It can calculate the probability for further malfunctions and issues recommendations for variable maintenance intervals.

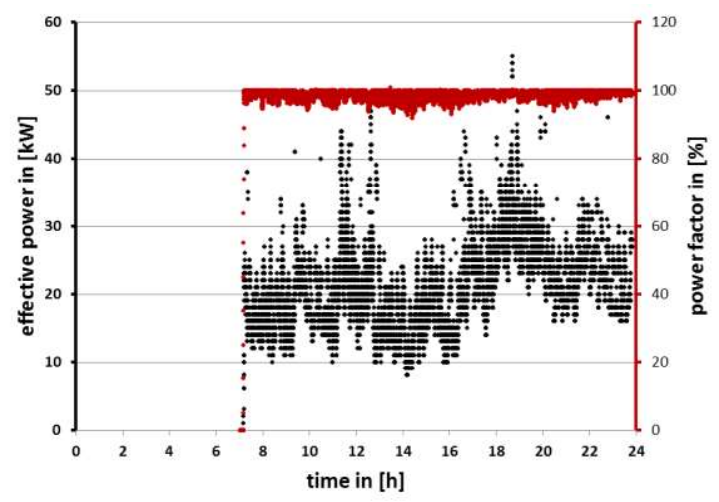

Fig.10. Effective power and power factor over a period of time

In addition to the previously described applications for power generators, these methods can equally be applied to power stores and distributors.

An additional application can calculate predictions on the expected life span of the power stores. If these are below the expected value, the operator can adjust the power store's utilisation to increase the life span.

Provided with additional sensor data, the application can create time-dependent distribution patterns for power distributors, from which the users' and departments' consumption pattern can be derived. Further improvements are to be developed.

\section{References}

[1] http://wiki.infowiss.net/Datenanalyse

[2] Werner A. Stahel, Statistische Datenanalyse, Eine Einführung für Naturwissenschaftler, 3. Auflage, p33ff 